\title{
Macroscopic entropy formulae and non-holomorphic corrections for supersymmetric black holes
}

\author{
Gabriel Lopes Cardoso ${ }^{\mathrm{a}}$, Bernard de Wit ${ }^{\mathrm{a}, \mathrm{b}}$, Thomas Mohaupt ${ }^{\mathrm{c}}$ \\ ${ }^{a}$ Institute for Theoretical Physics, Utrecht University, 3508 TA Utrecht, The Netherlands \\ ${ }^{\mathrm{b}}$ Max-Planck-Institut für Gravitationsphysik, Albert-Einstein-Institut, Am Mühlenberg 1, D-14476 \\ Golm, Germany \\ ${ }^{c}$ Martin-Luther-Universität Halle-Wittenberg, Fachbereich Physik, D-06099 Halle, Germany
}

Received 16 June 1999; accepted 8 September 1999

\begin{abstract}
In four-dimensional $N=2$ compactifications of string theory or M-theory, modifications of the Bekenstein-Hawking area law for black hole entropy in the presence of higher-derivative interactions are crucial for finding agreement between the macroscopic entropy obtained from supergravity and subleading corrections to the microscopic entropy obtained via state counting. Here we compute the modifications to the area law for various classes of black holes, such as heterotic black holes, stemming from certain higher-derivative gravitational Wilsonian coupling functions. We consider the extension to heterotic $N=4$ supersymmetric black holes and their type-II duals and we discuss its implications for the corresponding micro-state counting. In the effective field theory approach the Wilsonian coupling functions are known to receive non-holomorphic corrections. We discuss how to incorporate such corrections into macroscopic entropy formulae so as to render them invariant under duality transformations, and we give a concrete example thereof. (C) 2000 Elsevier Science B.V. All rights reserved.
\end{abstract}

PACS: 04.65.+e; 04.70.-s; 11.25.Pm

Keywords: Black holes; Entropy; Supersymmetry; String theory

\section{Introduction}

In the presence of higher-derivative interactions, modifications of the BekensteinHawking area law for the macroscopic entropy of a black hole are crucial for finding agreement with microscopic entropy calculations based on state counting. The latter involve corrections that are subleading in the limit of large charges, and these corrections are captured on the macroscopic side by the modifications of the area law that

E-mail addresses: cardoso@phys.uu.nl (G.L. Cardoso), bdewit@phys.uu.nl (B. de Wit), mohaupt@hera1.physik.uni-halle.de (T. Mohaupt). 
ensure the validity of the first law of black hole mechanics in the presence of higher-derivative interactions. For supersymmetric black hole solutions arising in fourdimensional $N=2$ supersymmetric effective field theories with terms quadratic in the Riemann tensor, they were determined in [1], where it was shown that, for a particular class of black holes arising in compactifications of M-theory and type-IIA string theory, the modified macroscopic entropy exactly matches the microscopic entropy computed in $[2,3]$.

The four-dimensional supersymmetric black hole solutions considered in [1] are static, rotationally symmetric solitonic interpolations between two $N=2$ supersymmetric ground states: flat Minkowski space-time at spatial infinity and Bertotti-Robinson space-time at the horizon [4]. The crucial ingredients of the work reported in [1] are as follows. One departs from the Bekenstein-Hawking area formula and adopts Wald's proposal for the entropy based on a Noether charge associated with an isometry evaluated at the corresponding Killing horizon [5-9]. The computation of the macroscopic entropy is based on the effective $N=2$ Wilsonian action describing the couplings of abelian vector multiplets to $N=2$ supergravity in the presence of a certain class of terms quadratic in the Riemann tensor. This Wilsonian action is determined from a holomorphic, homogeneous function $F(Y, \Upsilon)$, where the $Y^{I}$ denote the (rescaled) complex scalar fields which reside in the abelian vector multiplets, and where $\Upsilon$ denotes the (rescaled) square of the (auxiliary) anti-self-dual Lorentz tensor field $T^{a b i j}$ which resides in the Weyl multiplet. The macroscopic entropy of a static, supersymmetric black hole computed from the effective Wilsonian Lagrangian is then given by [1]

$$
\mathscr{S}_{\text {macro }}=\pi\left[|Z|^{2}+4 \operatorname{Im}\left(\Upsilon F_{\Upsilon}(Y, \Upsilon)\right)\right] \quad \text { with } \Upsilon=-64
$$

The first term in the entropy formula, $|Z|^{2}=p^{I} F_{I}(Y, \Upsilon)-q_{I} Y^{I}$, coincides with the Bekenstein-Hawking area contribution. The values of the scalar fields $Y^{I}$ on the horizon are, when assuming fixed-point behaviour [10-15], determined in terms of the magnetic and electric charges $\left(p^{I}, q_{I}\right)$ carried by the black hole. Their precise value is determined from a set of equations, called the stabilization equations, which take the following form [16]:

$$
Y^{I}-\bar{Y}^{I}=i p^{I}, \quad F_{I}(Y, \Upsilon)-\bar{F}_{I}(\bar{Y}, \bar{\Upsilon})=i q_{I} .
$$

The entropy is thus entirely determined in terms of the charges carried by the extremal black hole.

The above equations can usually not be solved in explicit form. Often they can be solved by iteration, order-by-order in $\Upsilon$. Let $Q$ denote a generic electric or magnetic charge. Then the homogeneity of the function $F$ implies that the entropy can be expanded in powers of $Q$, according to

$$
\mathscr{S}_{\text {macro }}=\pi \sum_{g=0}^{\infty} a_{g} Q^{2-2 g},
$$

with constant coefficients $a_{g}$. These coefficients follow from the coefficient functions in the power expansion for the holomorphic function $F$, which takes the form $F(Y, \Upsilon)=$ $\sum_{g \geqslant 0} F^{(g)}(Y) \Upsilon^{g}$.

In this paper we present a variety of applications of the entropy formula (1.1). In particular we consider $N=2$ heterotic black holes, whose macroscopic entropy formula can be generalized to the case of $N=4$ supersymmetric black holes. In order to 
appreciate the consequences of our results we will return to the counting of micro-states in the dual type-II or M-theory version of these black holes, following the same approach as in [2,3]. In this analysis certain special features of $K 3 \times T^{2}$ emerge which are not present for generic Calabi-Yau threefolds, implying that the micro-state counting in the former case is more subtle than in the latter case. Based on this analysis we propose a modification of the state counting for $K 3 \times T^{2}$ which is consistent with the macroscopic result.

Several comments are in order here. First, as mentioned above, the entropy formula (1.1) is based on the Wilsonian Lagrangian approach. In this approach, the coupling function multiplying the square of the Weyl tensor $C_{\mu \nu \rho \sigma}$ is denoted by $\operatorname{Im} F_{\gamma}$. It is customary to assume that $F_{\Upsilon}$ possesses a power expansion in $\Upsilon$, i.e. $F_{\Upsilon}=$ $\sum_{g \geqslant 1} F^{(g)}(Y) \Upsilon^{g-1}$. As pointed out recently in [17,18], this is not necessarily the case due to the fact that the $F_{\Upsilon}$ is non-analytic at $\Upsilon=0$. Second, the stabilization equations (1.2) are constructed out of the two symplectic vectors $\left(p^{I}, q_{I}\right)$ and $\left(Y^{I}, F_{I}(Y, \Upsilon)\right)$. It is important to note that under symplectic transformations the $Y^{I}$ will in general transform into $\Upsilon$-dependent terms. Thus, under the subset of duality transformations the moduli fields $z^{A}=Y^{A} / Y^{0}$ will, in the presence of $C_{\mu \nu \rho \sigma}^{2}$ terms (that is $F_{\Upsilon} \neq 0$ ), transform into $\Upsilon$-dependent quantities! Finally, we recall that in theories with massless fields the physical effective couplings are different from the Wilsonian couplings, which presuppose the presence of an infrared cut-off. While the physical couplings have different analyticity properties, they fully capture the physics and its underlying symmetries. Thus, in order to arrive at entropy formulae that are manifestly duality invariant, it is necessary to modify both (1.1) and (1.2), which were derived in a Wilsonian approach, by taking into account non-holomorphic corrections to the physical effective couplings. In the context of string theory one can actually determine these non-holomorphic terms, because the massless states are included in the string loops. Here we choose to incorporate the non-holomorphic corrections by imposing the correct symmetry behaviour. Therefore we include certain non-holomorphic corrections to the $\Upsilon$-dependent part of the function $F$, or equivalently, to $F_{\Upsilon}(Y, \bar{Y}, \Upsilon)$. Here the corresponding $F(Y, \bar{Y}, \Upsilon)$ is homogeneous of degree two in $Y$ and of degree zero in $\bar{Y}$. The corrections to $F_{\Upsilon}$ should be such that the associated symplectic vector $\left(Y^{I}, F_{I}(Y, \bar{Y}, \Upsilon)\right)$ transforms in the same way as $\left(p^{I}, q_{I}\right)$ under symplectic transformations associated with electric/magnetic duality. The associated stabilization equations now read

$$
Y^{I}-\bar{Y}^{I}=i p^{I}, \quad F_{I}(Y, \bar{Y}, \Upsilon)-\bar{F}_{I}(Y, \bar{Y}, \bar{\Upsilon})=i q_{I}
$$

Provided that the duality transformations on the $Y^{I}$ induce the corresponding symplectic transformations on the $F_{I}$, the quantity $|Z|^{2}=p^{I} F_{I}(Y, \bar{Y}, \Upsilon)-q_{I} Y^{I}$ is then invariant under duality transformations. However, in order to arrive at a duality invariant expression for the entropy of an $N=2$ black hole, it will not suffice to only take into account the above modification of $F_{\Upsilon}$ and, hence, of $|Z|^{2}$. As it turns out, also the second term associated with the deviation from the area law requires a non-holomorphic correction. Hence we are forced to use the following expression for the entropy:

$$
\begin{aligned}
& \mathscr{S}_{\text {macro }}=\pi\left[p^{I} F_{I}(Y, \bar{Y}, \Upsilon)-q_{I} Y^{I}+4 \operatorname{Im}\left(\Upsilon F_{\Upsilon}(Y, \bar{Y}, \Upsilon)+\Delta(Y, \bar{Y}, \Upsilon)\right)\right], \\
& \text { with } \Upsilon=-64,
\end{aligned}
$$


where $\Delta(Y, \bar{Y}, \Upsilon)$ denotes an appropriate quantity whose role is to render $\operatorname{Im}\left(\Upsilon F_{\Upsilon}+\Delta\right)$ duality invariant. In general it should be complicated to determine the explicit form of the non-holomorphic corrections entering both in (1.4) and (1.5) due to the fact, mentioned above, that the moduli fields $z^{A}$ will transform into $\Upsilon$-dependent terms under duality transformations. This is to be contrasted with the usual approach for determining non-holomorphic corrections to physical couplings in string theory [19-23], in which each of the gravitational coupling functions in the expansion of the holomorphic function $F$ in powers of $\Upsilon$ is required to transform as a modular function of the appropriate weight. This is achieved by taking into account non-holomorphic corrections to these coupling functions, which are governed by a set of recursive holomorphic anomaly equations. In the case at hand, however, not only does $F(Y, \bar{Y}, \Upsilon)$ and therefore also the symplectic vector $\left(Y^{I}, F_{I}(Y, \bar{Y}, \Upsilon)\right)$ and $|Z|^{2}$ receive non-holomorphic corrections, but there are additional non-holomorphic contributions encoded in $\Delta$. While this is an important feature of the above mechanism for incorporating non-holomorphic corrections into macroscopic entropy formulae, its real significance is not yet clear to us.

The structure of this paper is as follows. In Section 2 we compute the macroscopic entropy of a generic tree-level black hole in a heterotic $N=2$ compactification in the presence of a tree-level $C_{\mu \nu \rho \sigma}^{2}$ term. Subsequently we consider its extension to $N=4$ supersymmetric black holes. In Section 3 we then reconsider the $N=2$ and $N=4$ counting of micro-states in the dual type-II and M-theory formulations of the black holes. We show that the expression for the macroscopic $N=4$ entropy agrees with a microscopic entropy computation along the lines of [2,3] for a class of black holes occurring in dual type-II compactifications on $K 3 \times T^{2}$, provided that the micro-state counting for $K 3 \times T^{2}$ is suitably modified, as we discuss. In Section 4 we turn to the issue of non-holomorphic corrections to the macroscopic entropy. We discuss a heterotic-like $N=2$ model for which we are able to determine the non-holomorphic corrections to the entropy needed for obtaining a strong-weak coupling duality invariant expression for the entropy. And finally, in Section 5, we use the recent results of $[17,18]$ to compute corrections to the macroscopic entropy of certain type-IIA black holes stemming from $T^{a b i j}$-dependent higher-derivative interaction terms in the effective action.

\section{Heterotic tree-level black holes}

In the presence of an $(S+\bar{S}) C_{\mu \nu \rho \sigma}^{2}$ term in the effective Wilsonian action, where $S$ is the dilaton field, the tree-level holomorphic function $F(Y, \Upsilon)$ associated with a heterotic $N=2$ compactification on $K 3 \times T^{2}$ is given by

$$
F(Y, \Upsilon)=-\frac{Y^{1} Y^{a} \eta_{a b} Y^{b}}{Y^{0}}+c_{1} \frac{Y^{1}}{Y^{0}} \Upsilon .
$$

Here

$$
Y^{a} \eta_{a b} Y^{b}=Y^{2} Y^{3}-\sum_{a=4}^{n}\left(Y^{a}\right)^{2}, \quad a=2, \ldots, n,
$$


with real constants $\eta_{a b}$ and $c_{1}$. In a type-II dual picture, these constants can be related to geometrical properties of the $K 3$ fibration of the corresponding Calabi-Yau space. The dilaton field $S$ is defined by $S=-i Y^{1} / Y^{0}$.

Using (1.2) with $I=a$ one readily proves that the $Y^{a}$ are given by

$$
Y^{a}=\frac{1}{S+\bar{S}}\left[-\frac{1}{2} \eta^{a b} q_{b}+i \bar{S} p^{a}\right],
$$

where $\eta^{a b} \eta_{b c}=\delta_{c}^{a}$. Using the same equations once more one establishes

$$
\begin{aligned}
|Z|^{2} & =p^{I} F_{I}-q_{I} Y^{I} \\
& =i\left(\bar{Y}^{I} F_{I}-Y^{I} \bar{F}_{I}\right) \\
& =(S+\bar{S})\left(\bar{Y}^{a} \eta_{a b} Y^{b}+\frac{\bar{Y}^{0}}{Y^{0}}\left[-Y^{a} \eta_{a b} Y^{b}+c_{1} \Upsilon\right]+\text { h.c. }\right),
\end{aligned}
$$

as well as

$$
\begin{aligned}
q_{1} p^{0} & =-\left(Y^{0}-\bar{Y}^{0}\right)\left(F_{1}-\bar{F}_{1}\right) \\
& =\left(\frac{\bar{Y}^{0}}{Y^{0}}-1\right)\left[-Y^{a} \eta_{a b} Y^{b}+c_{1} \Upsilon\right]+\text { h.c. }
\end{aligned}
$$

Combining these two equations and substituting the values for the $Y^{a}$ yields

$$
|Z|^{2}=(S+\bar{S})\left(\frac{1}{2}\langle N, N\rangle+\left(c_{1} \Upsilon+\text { h.c. }\right)\right) \text {, }
$$

where $\langle N, N\rangle=2\left(N^{0} N^{1}+N^{a} \eta_{a b} N^{b}\right)=2\left(p^{0} q_{1}+p^{a} \eta_{a b} p^{b}\right)$. The vector $N^{I}$ of magnetic charges (in a certain duality basis) is defined by $N^{I}=\left(p^{0}, q_{1}, p^{2}, p^{3}, \ldots, p^{n}\right)$, and transforms linearly under the target-space duality group $\mathrm{SO}(2, n-1)$. The second independent vector of electric charges, which also transforms linearly under the targetspace duality group, will be needed shortly and equals $M_{I}=\left(q_{0},-p^{1}, q_{2}, q_{3}, \ldots, q_{n}\right)$.

From (2.6) we can now determine the entropy of a generic heterotic black hole, by using (1.1). The second term in the formula doubles the coefficient of the second term in (2.6) and we find

$$
\mathscr{S}_{\text {macro }}=\frac{1}{2} \pi(S+\bar{S})\left(\langle N, N\rangle-512 c_{1}\right) .
$$

For $c_{1}=0$ this expression coincides with the one given in [24], where it was also shown that in the presence of string-loop corrections the entropy takes the same form as the tree-level entropy, but with the heterotic tree-level coupling constant $S+\bar{S}$ replaced by the perturbative coupling constant.

Now we continue and find the equations that govern the value of the dilaton field in terms of the charges. Just as above we use Eqs. (1.2) to write down expressions for the combinations $S \bar{S} q_{1} p^{0}+q_{0} p^{1}$ and $i(\bar{S}-S) q_{1} p^{0}+q_{1} p^{1}-q_{0} p^{0}$, which do not explicitly depend on $Y^{0}$. This leads to two equations for $S$ :

$$
\begin{aligned}
& S \bar{S}\langle N, N\rangle=\langle M, M\rangle-2(S+\bar{S})\left(c_{1} \Upsilon S+\text { h.c. }\right), \\
& (S-\bar{S})\langle N, N\rangle=2 i M \cdot N+2(S+\bar{S})\left(c_{1} \Upsilon-\text { h.c. }\right) .
\end{aligned}
$$


At the horizon this leads to the following results for the dilaton and for the entropy formula:

$$
\begin{aligned}
& S=\sqrt{\frac{\langle M, M\rangle\langle N, N\rangle-(M \cdot N)^{2}}{\langle N, N\rangle\left(\langle N, N\rangle-512 c_{1}\right)}+i \frac{M \cdot N}{\langle N, N\rangle},} \\
& \mathscr{S}_{\text {macro }}=\pi \sqrt{\langle M, M\rangle\langle N, N\rangle-(M \cdot N)^{2}} \sqrt{1-\frac{512 c_{1}}{\langle N, N\rangle}},
\end{aligned}
$$

where we recall the three target-space duality invariant combinations of the charges [25],

$$
\begin{aligned}
& \langle M, M\rangle=2\left(M_{0} M_{1}+\frac{1}{4} M_{a} \eta^{a b} M_{b}\right)=2\left(-q_{0} p^{1}+\frac{1}{4} q_{a} \eta^{a b} q_{b}\right), \\
& \langle N, N\rangle=2\left(N^{0} N^{1}+N^{a} \eta_{a b} N^{b}\right)=2\left(p^{0} q_{1}+p^{a} \eta_{a b} p^{b}\right), \\
& M \cdot N=M_{I} N^{I}=q_{0} p^{0}-q_{1} p^{1}+q_{2} p^{2}+\ldots+q_{n} p^{n} .
\end{aligned}
$$

A significant feature of these results is that they are manifestly invariant under the continuous $\mathrm{SO}(2, n-1)$ target-space duality transformations, which are valid in the tree approximation. We analyze these transformations as well as the discrete $S$-duality transformations in Section 4.

The above entropy formula can be immediately generalized to the case of a heterotic $N=4$ compactification with a tree-level term proportional to $(\operatorname{Re} S) C_{\mu \nu \rho \sigma}^{2}$ turned on. This can be done by replacing the $\mathrm{SO}(2, n-1)$ bilinear combinations of the charges by the appropriate $\mathrm{SO}(6,22)$ bilinears in $(2.9)$. Here we remind the reader that the $N=4$ electric/magnetic charges are associated with six graviphotons of pure supergravity and 22 vector multiplets, whose $N=2$ decomposition is as follows. One graviphoton belongs to the $N=2$ graviton multiplet, four graviphotons belong to the two $N=2$ gravitini multiplets (which cannot be incorporated in the context of the $N=2$ theory discussed above), and one graviphoton belongs to an $N=2$ vector multiplet. The additional $22 N=4$ matter multiplets each decompose into an $N=2$ vector and an $N=2$ hypermultiplet, so that altogether one is dealing with 67 complex moduli. In the $N=2$ truncation one thus has $n=23$ and must suppress 4 of the 28 electric and 4 of the 28 magnetic charges. Of course, the $N=2$ truncation does not constitute by itself a heterotic $N=2$ string compactification, which has only $n=19$ and which is described by different Wilsonian couplings functions beyond the tree approximation.

The $N=4$ supersymmetric heterotic models have dual realizations as type-II string theory compactified on $K 3 \times T^{2}$ and as eleven-dimensional M-theory compactified on $K 3 \times T^{2} \times S^{1}$. The $N=2$ models have dual realizations as compactifications of the same theories but now on a generic Calabi-Yau manifold. In Section 3 we will discuss certain aspects of the state counting for these dual realizations.

\section{State counting for type-II and M-theory black holes}

Motivated by the possible extension of the heterotic $N=2$ black hole entropy formula to the corresponding case of $N=4$ supersymmetric black holes, we now return to the counting of micro-states for the dual type-II and M-theory realizations of the 
heterotic models discussed above. If one restricts the four-dimensional charges in an appropriate way (we take $p^{0}=q_{A}=0$ for convenience, but the discussion should also apply to the more general case $p^{0}=0$ by replacing $q_{0}$ by $\hat{q}_{0}$ as in [2]), then the black hole can be given a ten- or eleven-dimensional interpretation, as follows. In the type-IIA picture one wraps a D4-brane on a holomorphic four-cycle $P$ in either a generic Calabi-Yau threefold or in $K 3 \times T^{2}$ and considers a bound state with $\left|q_{0}\right|$ D0-branes. This will then lead to a four-dimensional black hole with $N=2$ or $N=4$ supersymmetry, respectively. In the M-theory picture one wraps an M5-brane on the same cycle $P$ and obtains a five-dimensional black string which is then wrapped around the M-theory circle. Adding $\left|q_{0}\right|$ quanta of lightlike momentum along this string yields a four-dimensional black hole with a finite event horizon.

In both pictures a microscopic entropy can be computed along the lines of [2,3]. The approach of Ref. [2] is based on analyzing the two-dimensional $\sigma$-model that describes the massless excitations of an M5-brane wrapped on $P$. Then, using Cardy's formula, the asymptotic density of states of a two-dimensional conformal field theory in a finite volume is given by

$$
d\left(\left|q_{0}\right|, c_{L}\right) \approx \exp \left(\mathscr{S}_{\text {micro }}\right) \approx \exp \left(2 \pi \sqrt{\frac{1}{6}\left|q_{0}\right| c_{L}}\right),
$$

where $\left|q_{0}\right|$ is large and counts quanta of lightlike, left-moving momentum, and $c_{L}$ is the central charge for the left-moving sector. On the other hand in Ref. [3] the asymptotic growth of the dimension of the moduli space of a bound state of a wrapped D4-brane with $\left|q_{0}\right|$ D0-branes was computed. The explicit formulae given in [2,3] assume certain properties of the four-cycle $P$, and there it was mentioned that in the case of $K 3 \times T^{2}$ additional subleading terms are to be expected.

The holonomy group of $K 3 \times T^{2}$ is $\mathrm{SU}(2)$, unlike the holonomy group of a generic Calabi-Yau threefold which is equal to SU(3). This difference reflects itself in the number of residual supersymmetries and in the different Hodge diamonds which we will discuss below. We will apply the methods of [2,3] in parallel to both a generic Calabi-Yau threefold and to $K 3 \times T^{2}$. As it will turn out, in the case of $K 3 \times T^{2}$ certain complications arise which indicate that the associated state counting has to be modified in order to achieve consistency with the macroscopic analysis based on $N=2$ supergravity.

Let us first recall some geometric properties of Calabi-Yau threefolds [26-29], starting with the homology and cohomology of a Calabi-Yau threefold $X$, which is either $K 3 \times T^{2}$ or a generic one and which corresponds to an $N=4$ or $N=2$ supersymmetric compactification, respectively. In the case of a generic Calabi-Yau manifold the only undetermined Hodge numbers are $h_{2,1}(X)$ and $h_{1,1}(X)$. In addition one has non-vanishing $h_{0,0}(X)=h_{3,0}(X)=1$, whereas all other independent Hodge numbers vanish.

The Hodge numbers of $K 3 \times T^{2}$ are easily obtained from those of $K 3$ and $T^{2}$. For later convenience let us derive them in terms of cohomology, i.e. by listing harmonic ( $p, q)$-forms. The harmonic forms on $T^{2}$ can be built out of the constant function, the $(1,0)$-form $d z$ and the $(0,1)$-form $d \bar{z}$, where $z$ is a holomorphic coordinate. On $K 3$ one has the constant function, no one-forms and 22 two-forms $\omega_{a}, a=2, \ldots, 23$, which can be decomposed into one (2,0)-form $\omega$, one $(0,2)$-form $\bar{\omega}$ and twenty $(1,1)$-forms. The reason for leaving out the index $a=1$ will become clear shortly. The harmonic 
( $p, q)$-forms on $K 3 \times T^{2}$ are readily found by taking appropriate products. The resulting independent Hodge numbers for $K 3 \times T^{2}$ are: $h_{0,0}=h_{1,0}=h_{2,0}=h_{3,0}=1$ and $h_{1,1}=h_{2,1}=21$. The two-forms on $K 3 \times T^{2}$ will be denoted by $\omega_{A}$ with $A=$ $1,2, \ldots, 23$, where $\omega_{1} \propto d z \wedge d \bar{z}$ and the $\omega_{a}$ are the $K 3$ forms introduced above.

The triple intersection numbers $C_{A B C}$ of a Calabi-Yau threefold are defined by

$$
C_{A B C}=\int_{X} \omega_{A} \wedge \omega_{B} \wedge \omega_{C}
$$

As we will be dealing with the integer-valued cohomology group $H^{2}(X, \mathbb{Z})$, the basic two-forms are normalized such that the intersection numbers are integers. For $X=K 3$ $\times T^{2}$ the appropriate normalization is based on

$$
\int_{K 3} \omega \wedge \bar{\omega}=1, \quad \int_{T^{2}} \omega_{1}=1, \quad \int_{K 3} \omega_{a} \wedge \omega_{b}=C_{a b}
$$

where $C_{a b}$ is the intersection form of two-cycles in $K 3$. The non-vanishing components of the triple intersection form $C_{A B C}$ of $K 3 \times T^{2}$ are therefore

$$
C_{a b 1}=C_{a b} .
$$

In order to discuss the wrapping of D4- or M5-branes on four-cycles, we turn to the homology. We recall that Poincaré duality provides a natural relation between $i$-forms $\omega$ and $2 k-i$ cycles $\Omega$ for every real $2 k$-fold $X$ by

$$
\int_{\Omega} \phi=\int_{X} \omega \wedge \phi
$$

for all $(2 k-i)$-forms $\phi$. This defines an isomorphism between $H^{i}(X, \mathbb{R})$ and $H_{2 k-i}(X, \mathbb{R})$. In the particular case of Calabi-Yau threefolds, four-cycles are dual to two-forms. We can therefore fix a basis for $H_{4}(X, \mathbb{R})$ by taking the four-cycles dual to a basis of $H^{2}(X, \mathbb{R})$. This basis will be denoted by $\Sigma_{A}$, where $A=1, \ldots b_{2}(X)$ and where $b_{2}(X)=\operatorname{dim}_{\mathbb{R}} H^{2}(X, \mathbb{R})=h_{2,0}(X)+h_{1,1}(X)+h_{0,2}(X)$ is the second Betti number. In the case of $X=K 3 \times T^{2}$ we have $b_{2}=23$ and the cycles $\Sigma_{A}$ with $A \neq 1$ are products of the basic two-cycles of $K 3$ with $T^{2}$, whereas $\Sigma_{1}$ corresponds to $K 3$. The four-cycle $P$ on which the D4- or M5-brane is wrapped can thus be expanded in a homology basis $\Sigma_{A}$,

$$
P=p^{A} \Sigma_{A}
$$

The integers $p^{A}$ count how many times the D4- or M5-brane is wrapped around the corresponding cycle, so that we are actually dealing with integer-valued cohomology and homology groups, $H^{2}(X, \mathbb{Z})$ and $H_{4}(X, \mathbb{Z})$. In four-dimensional terms the $p^{A}$ are magnetic charges, which are quantized according to Dirac's rule.

By Poincaré duality the triple intersection numbers also count the numbers (weighted with orientation) of intersections of generic four-cycles in a given homology class. The triple intersection for a four-cycle is therefore

$$
P \cdot P \cdot P=C_{A B C} p^{A} p^{B} p^{C} .
$$


For $K 3 \times T^{2}$ the non-vanishing triple intersections of four-cycles are

$$
\Sigma_{a} \cdot \Sigma_{b} \cdot \Sigma_{1}=C_{a b},
$$

so that the triple intersection of a four-cycle $P$ in the class $p^{a} \Sigma_{a}+p^{1} \Sigma_{1}$ is given by

$$
P \cdot P \cdot P=3 C_{a b} p^{a} p^{b} p^{1} .
$$

Another quantity that will appear in the microscopic entropy formula is the second Chern class $c_{2}(X)$ of $X$ evaluated on the four-cycle $P$. Recall that the second Chern class is a four-form which defines a cohomology class in $H^{4}(X, \mathbb{Z})$. Hence there is a dual two-cycle in $H_{2}(X, \mathbb{Z})$ which we will denote be $C_{2}$. Since $X$ is a Calabi-Yau manifold we can choose a basis $\Sigma^{A}$ of $H_{2}(X, \mathbb{Z})$ which is dual to the basis $\Sigma_{A}$ of $H_{4}(X, \mathbb{Z})$, i.e. $\Sigma^{A} \cdot \Sigma_{B}=\delta_{B}^{A}$. Expanding $C_{2}=c_{2 A} \Sigma^{A}$ we find

$$
C_{2} \cdot P=\int_{P} c_{2}(X)=c_{2 A} p^{A} .
$$

For $K 3 \times T^{2}$ the total Chern class can be computed from the total Chern classes of $K 3$ and $T^{2}$ by the Whitney product formula

$$
c\left(K 3 \times T^{2}\right)=c(K 3) \wedge c\left(T^{2}\right)=\left(1+c_{2}(K 3)\right) \wedge 1=1+c_{2}(K 3),
$$

where we used $c_{1}(K 3)=0$ and $c_{1}\left(T^{2}\right)=0$. Since $c_{2}(K 3)$ is the Euler class of $K 3$ and since $K 3$ has Euler number 24 we find

$$
C_{2} \cdot P=\chi(K 3) p^{1}=24 p^{1} .
$$

So far we have discussed only topological aspects of the threefold. But in order to get a supersymmetric state by wrapping a D4- or M5-brane on $P$, the four-cycle must be holomorphic [30] and must therefore define a divisor in $X$. A divisor is an integer linear combination of irreducible analytic hypersurfaces, where the word analytic emphasizes that a divisor can be locally characterized as the zero locus of a holomorphic function. The corresponding homology classes are called algebraic. According to the Lefschetz theorem on $(1,1)$-classes, every cohomology class in $H^{2}(X, \mathbb{Z}) \cap H^{1,1}(X, \mathbb{R})$ is algebraic. Here the integer valued cohomology group $H^{2}(X, \mathbb{Z})$ might be visualized as a lattice in the real vector space $H^{2}(X, \mathbb{R})$, whereas $H^{1,1}(X, \mathbb{R})$ is a linear subspace. If the dimension of $H^{1,1}(X, \mathbb{R})$ is smaller than the dimension of $H^{2}(X, \mathbb{R})$ and if the subspace $H^{1,1}(X, \mathbb{R})$ is generic, then it will intersect the lattice $H^{2}(X, \mathbb{Z})$ in no point but the origin. Therefore it is in general not guaranteed that algebraic classes exist.

For generic Calabi-Yau threefolds, $h_{2,0}(X)=0$, and therefore $H^{1,1}(X, \mathbb{R})$ will coincide with $H^{2}(X, \mathbb{R})$, implying that all fourth homology classes are algebraic. However, for $K 3 \times T^{2}$ we have $h_{2,0}=1$ and therefore $H^{1,1}(X, \mathbb{R})$ is only contained in, but not identical with $H^{2}(X, \mathbb{R})$. Obviously the problem resides in the $K 3$ factor, which has $h_{2,0}(K 3)=1$. Whether or not a $K 3$ space has algebraic classes is controlled by the so-called algebraic lattice, or Picard lattice of $K 3$,

$$
\Gamma_{P}=H^{2}(K 3, \mathbb{Z}) \cap H^{1,1}(K 3, \mathbb{R}) .
$$

Special $K 3$ spaces, which have a non-empty $\Gamma_{P}$ and therefore have holomorphic cycles, are called algebraic. Hence we will below restrict ourselves to these algebraic $K 3$ 
surfaces, which are associated to subspaces of codimension 1 in the moduli space of complex structures. ${ }^{1}$ The computation of the microscopic entropy amounts to counting the massless excitations of a D4- or M5-brane wrapped on $P$. In addition to being holomorphic, one has to require that $P$ is a so-called 'very ample' divisor in order to ensure that the entropy calculation is not affected by $\alpha^{\prime}$-corrections in the type-II picture and by instanton corrections in the M-theory picture, as we will discuss in due course. By definition a divisor $P$ in a space $X$ is called very ample if $X$ can be embedded into a complex projective space $\mathbb{C P}^{N}$ such that $P$ is the intersection of $X$ with some hyperplane in $\mathbb{C P}^{N}$. Then Bertini's theorem implies that, generically, $P$ is a smooth manifold. In our particular case the divisor $P$ is a compact, complex Kähler surface. On general grounds one therefore expects that the collective modes of the wrapped brane are controlled by the Hodge numbers $h_{p, q}(P)$ of $P$. Therefore we will discuss how the independent Hodge numbers $h_{1,0}(P), h_{2,0}(P)$ and $h_{1,1}(P)$ of $P$ are related to known quantities of $X$.

Three standard invariants can be computed using index theorems (only two of them are independent due to the fact that a complex surface can only have two independent Chern classes). These are the Euler number $\chi$, the holomorphic Euler number $\chi_{h}$ and the Hirzebruch signature $\sigma$, defined by

$$
\begin{aligned}
& \chi(P)=\sum_{p, q}(-1)^{p+q} h_{p, q}(P)=2-4 h_{1,0}(P)+2 h_{2,0}(P)+h_{1,1}(P), \\
& \chi_{h}(P)=\sum_{q}(-1)^{q} h_{0, q}(P)=1-h_{1,0}(P)+h_{2,0}(P) \\
& \sigma(P)=b_{2}^{+}(P)-b_{2}^{-}(P)=2 h_{2,0}(P)-h_{1,1}(P)+2,
\end{aligned}
$$

where we used that $b_{2}^{ \pm}(P)$, which denote the number of (anti)self-dual harmonic two-forms, are equal to $b_{2}^{+}(P)=2 h_{2,0}(P)+1$ and $b_{2}^{-}(P)=h_{1,1}(P)-1$ for a compact Kähler surface. Now $\chi(P)$ and $\sigma(P)$ can be computed using the following index theorems:

$$
\chi(P)=\int_{P} c_{2}(P), \quad \sigma(P)=\int_{P}\left(-\frac{2}{3} c_{2}(P)+\frac{1}{3} c_{1}(P)^{2}\right)
$$

which then determines $\chi_{h}(P)=\frac{1}{4}(\chi+\sigma)$ as well.

To every very ample divisor $P$ in $X$ one can associate a line bundle $L$ over $X$, such that $P$ is the zero locus of a holomorphic section of $L$. The space $H^{0}(X, L)$ of holomorphic sections of $L$ is closely related to the moduli space $\mathscr{M}(P)$ of $P$, i.e. to the space of deformations of $P$ inside $X$. Since divisors are obtained as zero loci of sections, multiplying a section by a non-vanishing complex number does not change the

\footnotetext{
${ }^{1}$ Conformal field theories describing type-II strings in the background of such algebraic $K 3$ spaces have been discussed in [31]. In particular the moduli spaces of algebraic $K 3$ string backgrounds factorize into two subspaces containing the complex structure moduli and the complexified Kähler moduli, respectively. If one compactifies type-II strings on $K 3 \times T^{2}$, then this is the part of the moduli space that can be described in an $N=2$ truncation of the $N=4$ theory.
} 
divisor. Therefore the moduli space of $P$ is the projective space associated to the vector space $H^{0}(X, L)$ :

$$
\mathscr{M}(P)=P H^{0}(X, L) .
$$

Another way of thinking about the moduli of $P$ is as sections of the normal bundle $N_{P / X}$ of $P$,

$$
N_{P / X}=\left.\mathscr{T} X\right|_{P} / \mathscr{T} P
$$

where $\left.\mathscr{T} X\right|_{P}$ is the holomorphic tangent bundle of $X$ restricted to $P$ and $\mathscr{T} P$ is the holomorphic tangent bundle of $P$. When wrapping a D-brane or M-brane on a holomorphic cycle, the scalar fields of the world volume theory corresponding to transverse motions of $P$ inside $X$ become sections of the normal bundle [32]. Both descriptions of the moduli of $P$ are equivalent because the first adjunction formula implies that the line bundles $N_{P / X}$ and $L$ are isomorphic.

The next step is to express the Chern classes of $P$ in terms of the Chern classes of $X$. Because the normal bundle $N_{P / X}$ of $P$ is the quotient of the tangent bundle of $X$ and of the tangent bundle of $P$, the Whitney product formula for Chern classes implies $c(X)=c(P) \wedge c\left(N_{P / X}\right)$ or

$$
1+c_{1}(X)+c_{2}(X)+\ldots=\left(1+c_{1}(P)+c_{2}(P)\right) \wedge\left(1+c_{1}\left(N_{P / X}\right)\right) .
$$

Since $X$ is Calabi-Yau, we have $c_{1}(X)=0$. Hence one gets the relations $c_{1}(P)+$ $c_{1}\left(N_{P / X}\right)=0$ and $c_{2}(P)+c_{1}(P) \wedge c_{1}\left(N_{P / X}\right)=c_{2}(X)$, which can be combined into

$$
c_{2}(P)=c_{2}(X)+c_{1}\left(N_{P / X}\right)^{2} .
$$

What remains is to express $c_{1}\left(N_{P / X}\right)$ by a quantity defined on $X$. To do so we recall that the first adjunction formula implies that the normal bundle $N_{P / X}$ is isomorphic to the line bundle $L$. In particular the first Chern classes of both line bundles are equal, $c_{1}\left(N_{P / X}\right)=c_{1}(L)$. Moreover the two-form $c_{1}(L)$ is Poincaré dual to the four-cycle $P$, and one can rewrite the above integrals as integrals over $X$ :

$$
\begin{aligned}
& \chi(P)=\int_{X}\left(c_{2}(X)+c_{1}(L)^{2}\right) \wedge c_{1}(L), \\
& \sigma(P)=\int_{X}\left(-\frac{2}{3} c_{2}(X)-\frac{1}{3} c_{1}(L)^{2}\right) \wedge c_{1}(L) .
\end{aligned}
$$

Finally the integrals on the right-hand side can be interpreted as intersection products using

$$
P^{3}:=P \cdot P \cdot P=\int_{X} c_{1}(L)^{3}, \quad \int_{P} c_{2}(X)=\int_{X} c_{1}(L) \wedge c_{2}(X)=C_{2} \cdot P,
$$

where $C_{2}$ is the two-cycle Poincaré dual to $c_{2}(X)$. With these results one can express the invariants in terms of intersection numbers,

$$
\chi(P)=P^{3}+C_{2} \cdot P, \quad \sigma(P)=-\frac{1}{3} P^{3}-\frac{2}{3} C_{2} \cdot P .
$$


Now it is straightforward to obtain the following relations for the Hodge numbers:

$$
\begin{aligned}
& h_{2,0}(P)=\frac{1}{6} P^{3}+\frac{1}{12} C_{2} \cdot P+h_{1,0}(P)-1, \\
& h_{1,1}(P)=\frac{2}{3} P^{3}+\frac{5}{6} C_{2} \cdot P+2 h_{1,0}(P),
\end{aligned}
$$

or, equivalently,

$$
\begin{aligned}
& b_{2}^{+}(P)=\frac{1}{3} P^{3}+\frac{1}{6} C_{2} \cdot P+2 h_{1,0}(P)-1, \\
& b_{2}^{-}(P)=\frac{2}{3} P^{3}+\frac{5}{6} C_{2} \cdot P+2 h_{1,0}(P)-1 .
\end{aligned}
$$

What remains is to calculate $h_{1,0}(P)$. Here one uses again that $P$ is a very ample divisor. Therefore the Lefschetz hyperplane theorem implies that $h_{1,0}(P)=h_{1,0}(X)$. Thus we have $h_{1,0}(P)=0$ when $X$ is a generic Calabi-Yau threefold and $h_{1,0}(P)=1$ when $X=K 3 \times T^{2}$.

The final step in the approach of [2] is to count the massless left-moving bosons and fermions of the wrapped M5-brane and to express them in terms of the topological quantities computed above. As argued in [3] the analysis of the equivalent D4-D0 system should give the same answer. We will follow Ref. [2].

In flat space, the massless degrees of freedom of the M5-brane correspond to a six-dimensional $N=(0,2)$ tensor multiplet, which contains a self-dual antisymmetric tensor, five scalars which describe transverse motions and two Weyl spinors. In order to compute the microscopic entropy one needs to dimensionally reduce this system on the divisor $P$ and to count the massless modes in the non-supersymmetric, left-moving sector of the resulting two-dimensional theory.

Dimensional reduction of the antisymmetric tensor on $P$ gives $b_{2}^{-}(P)$ left-moving and $b_{2}^{+}(P)$ right-moving scalars together with $b_{1}(P)=2 h_{1,0}(P)$ gauge fields. Two-dimensional gauge fields do not carry dynamical degrees of freedom themselves, but may modify the counting for the other modes. For a generic threefold one has $b_{1}(P)=b_{1}(X)$ $=0$ and this problem is absent. A full analysis would require a detailed study of the effective $\sigma$-model describing the collective modes of a M5-brane wrapped on the divisor $P$ in $K 3 \times T^{2}$. This will not be attempted here.

Instead we will first review the counting performed in [2,3] and assume that there are no modifications for $K 3 \times T^{2}$. As we will see in a moment the resulting microscopic formula contains, in the case of $K 3 \times T^{2}$, sub-subleading terms that are puzzling from the supergravity point of view. Moreover, with this unmodified mode counting the right-moving sector of the effective M5-brane theory turns out not to be supersymmetric. This implies that the microscopic analysis in the case of $K 3 \times T^{2}$ must be more subtle. We will then propose a modification of the zero-mode counting which is consistent with the supergravity analysis.

The M5-brane theory in flat space has five scalars describing the transverse motions. These split into three scalars corresponding to the position of the brane in the three non-compact space dimensions and two scalars corresponding to motions of the divisor $P$ inside the Calabi-Yau threefold. Whereas the first set just gives three scalar zero-modes, the zero-modes associated to the second set are more subtle. One gets one zero-mode for every independent holomorphic deformation of $P$ inside $X$. In other words these zero-modes are sections of the normal bundle $N_{P / X}$. As reviewed above the dimension of the corresponding moduli space is

$$
\operatorname{dim}_{\mathbb{R}} \mathscr{M}(P)=\operatorname{dim}_{\mathbb{R}} P H^{0}(X, L)=\operatorname{dim}_{\mathbb{R}} H^{0}(X, L)-2 .
$$


It was emphasized in [2] that this quantity is hard to compute in general, but that there is a Riemann-Roch index theorem for the holomorphic Euler number $\chi_{h}(L)=$ $\sum_{i}(-1)^{i} \operatorname{dim}_{\mathbb{C}} H^{i}(X, L)$ of line bundles $L$ over complex manifolds. We already mentioned that the divisor $P$ is chosen to be very ample. Then the Kodaira vanishing theorem together with the fact that $X$ is a Calabi-Yau manifold implies that $\operatorname{dim}_{\mathbb{C}} H^{i}(X, L)=0$ for $i>0$. Therefore $\operatorname{dim}_{\mathbb{R}} \mathscr{M}(P)=2 \chi_{h}(P)-2$ and hence

$$
\operatorname{dim}_{\mathbb{R}} \mathscr{M}(P)=2\left[h_{2,0}(P)-h_{1,0}(P)\right] .
$$

This is the number of real left- and right-moving scalars related to transverse motions of $P$ inside $X$. Combining it with the counting of modes descending from the antisymmetric tensor field, one finds that the number of left- and right-moving bosonic degrees of freedom is

$$
\begin{aligned}
& N_{\text {bosonic }}^{\text {left }}=\operatorname{dim}_{\mathbb{R}} \mathscr{M}(P)+3+b_{2}^{-}(P)=2 h_{2,0}(P)+h_{1,1}(P)+2-2 h_{1,0}(P), \\
& N_{\text {bosonic }}^{\text {right }}=\operatorname{dim}_{\mathbb{R}} \mathscr{M}(P)+3+b_{2}^{+}(P)=4 h_{2,0}(P)+4-2 h_{1,0}(P) .
\end{aligned}
$$

The number of real left- and right-moving fermions, on the other hand, is given by the sum of odd and even cohomology elements, respectively [3],

$$
N_{\text {fermionic }}^{\text {left }}=4 h_{1,0}(P), \quad N_{\text {fermionic }}^{\text {right }}=4\left[h_{2,0}(P)+h_{0,0}(P)\right] .
$$

The effective two-dimensional theory describing the collective modes of a BPS black hole is a $(0,4)$ supersymmetric sigma-model. Here we recall that the $(0,4)$ world-sheet supersymmetry is crucial for describing an $N=2$ BPS black hole in four-dimensional space-time. Therefore, the number of right-moving bosons and fermions has to match. Moreover the right-moving scalars are expected to parametrize a quaternionic manifold and therefore the number of right-moving real bosons should be a multiple of four. Inspection of (3.27) and (3.28) shows that in the case of a generic threefold, for which $h_{1,0}(P)=0$, the counting of right-moving modes is consistent with $(0,4)$ supersymmetry, whereas this is not the case for $K 3 \times T^{2}$, for which $h_{1,0}(P)=1$. This implies that the zero-mode counting for $K 3 \times T^{2}$ has to deviate from the one described above.

Using (3.27) and (3.28) the central charge of the left-moving sector is computed to be

$$
\begin{aligned}
c_{L} & =N_{\text {bosonic }}^{\text {left }}+\frac{1}{2} N_{\text {fermionic }}^{\text {left }}=P^{3}+C_{2} \cdot P+4 h_{1,0}(P) \\
& =C_{A B C} p^{A} p^{B} p^{C}+c_{2 A} p^{A}+4 h_{1,0}(P) .
\end{aligned}
$$

For the generic case, where $h_{1,0}(P)=0$, this leads via Cardy's formula to the final result $[2,3]$

$$
\mathscr{S}_{\text {micro }}=2 \pi \sqrt{\frac{1}{6}\left|q_{0}\right|\left(C_{A B C} p^{A} p^{B} p^{C}+c_{2 A} p^{A}\right)} .
$$

In the case of $K 3 \times T^{2}$ the intersection and second Chern class numbers take a simpler form. Using (3.29) with $h_{1,0}(P)=1$, the associated microscopic entropy (3.1) is computed to be

$$
\mathscr{S}_{\text {micro }}=2 \pi \sqrt{\frac{1}{6}\left|q_{0}\right|\left(3 C_{a b} p^{a} p^{b} p^{1}+24 p^{1}+4\right)} .
$$

We note that the sub-subleading third term in this expression is not consistent with the macroscopic computation of the entropy based on $N=2$ supergravity. As mentioned in the introduction, the homogeneity properties of the prepotential together with the stabilization equations imply that the entropy should take the form (1.3), and hence terms containing odd powers of the charges cannot be present in the entropy formula. 
Another reason why this sub-subleading term is troublesome is that the approaches of [2] and [3] seem to give a slightly different numerical value for this term. Namely, when substituting the Hodge numbers of $P$ into the entropy formula [3]

$$
\mathscr{S}_{\text {micro }}=2 \pi \sqrt{\frac{1}{6}\left|q_{0}\right|\left(b_{\text {even }}(P)+\frac{1}{2} b_{\text {odd }}(P)\right)},
$$

where $b_{\text {even }}(P)$ and $b_{\text {odd }}(P)$ denote the sums of the even and of the odd Betti numbers, respectively, we obtain (3.31) but with a six instead of a four in the last term. Note that for generic threefolds, where $h_{1,0}(P)=0$, both approaches [2,3] yield (3.30).

How is the zero-mode counting for $K 3 \times T^{2}$ to be modified in order to remove the inconsistencies mentioned above? Let us recall that there are $b_{1}=2 h_{1,0}(P)=2$ non-dynamical gauge fields present. If we assume that the zero-modes are charged and couple to these gauge fields, then the following mechanism suggests itself. Due to gauge invariance, the number of left- and right-moving scalar fields is reduced by two, so that the number of right-moving scalar fields is indeed a multiple of four. Due to supersymmetry this must be accompanied by the removal of four right-moving fermionic real degrees of freedom. If, in addition, we assume that the removal of fermionic degrees of freedom is left-right symmetric, then the actual number of left-moving fermionic degrees of freedom is zero. The central charge in the left-moving sector is now computed to be $c_{L}=P^{3}+C_{2} \cdot P=3 C_{a b} p^{a} p^{b} p^{1}+24 p^{1}$, which is odd in the charges. The resulting microscopic entropy formula is then in full agreement with the macroscopic computation, which we now briefly describe.

Let us analyze the same system from the supergravity point of view. The Wilsonian action controlling the vector multiplet sector of an $N=2$ compactification and the relevant $N=2$ subsector of an $N=4$ compactification is encoded in the holomorphic function

$$
\begin{aligned}
F(Y, \Upsilon) & =-\frac{1}{6} C_{A B C} \frac{Y^{A} Y^{B} Y^{C}}{Y^{0}}-\frac{1}{24} \frac{1}{64} c_{2 A} \frac{Y^{A}}{Y^{0}} \Upsilon \\
& =-\frac{1}{2} C_{a b} \frac{Y^{1} Y^{a} Y^{b}}{Y^{0}}-\frac{1}{64} \frac{Y^{1}}{Y^{0}} \Upsilon,
\end{aligned}
$$

where the first formula refers to the generic case, whereas the second formula refers to a compactification on $K 3 \times T^{2}$. In the latter case $Y^{a} / Y^{0}$ are the complexified Kähler moduli of an algebraic $K 3$ and $Y^{1} / Y^{0}$ is the complexified Kähler modulus of $T^{2}$.

Since the function (3.33) represents the leading part of the holomorphic function in the $\alpha^{\prime}$-expansion, ${ }^{2}$ we briefly discuss under which conditions a solution based on this function can be reliable [2,24]. The issue of $\alpha^{\prime}$-corrections is not quite the same for compactifications on generic threefolds and for compactifications on $K 3 \times T^{2}$. For generic threefolds, which lead to $N=2$ compactifications, both the prepotential $F(Y, \Upsilon$ $=0$ ) and the $C^{2}$-coupling function $F_{\Upsilon}$ receive $\alpha^{\prime}$-corrections. In contrast the metric on the $N=4$ moduli space and, hence, the prepotential $F(Y, \Upsilon=0)$ in the $N=2$ subsector of an $N=4$ compactification, do not receive $\alpha^{\prime}$-corrections. However, the gravitational $C^{2}$-coupling function $F_{\Upsilon}$ does get corrected [33]. Therefore (3.33) is valid for both $N=2$ and $N=4$ compactifications in the limit of large Kähler moduli, only. In

\footnotetext{
${ }^{2}$ In the M-theory picture $\alpha^{\prime}$-corrections appear as instanton corrections corresponding to wrapped M-branes. For convenience we will use the type-IIA picture in the following.
} 
particular the Kähler moduli $\operatorname{Im}\left(Y^{A} / Y^{0}\right)$ must take large positive values at the event horizon. Since the Kähler moduli are space dependent for a generic extremal four-dimensional black hole solution, the geometry of the internal manifold will change accordingly over space. The values of the moduli at the event horizon are fixed by the stabilization equations. For a black hole with non-vanishing charges $q_{0}, p^{A}$ we have [1]

$$
\left.\frac{Y^{A}}{Y^{0}}\right|_{\text {horizon }}=i p^{A} \sqrt{\frac{6\left|q_{0}\right|}{C_{B C D} p^{B} p^{C} p^{D}+c_{2 B} p^{B}}} .
$$

Thus, in order to guarantee $\operatorname{Im} Y^{A} /\left.Y^{0}\right|_{\text {horizon }} \gg 0$ we need to impose $\left|q_{0}\right| \gg p^{A}>0$. In fact we will impose the stronger condition $\left|q_{0}\right| \gg p^{A} \gg 0$ in order to make contact with the microscopic entropy counting.

From the microscopic point of view the charges $p^{A}$ are the expansion coefficients of the divisor $P$ in the homology basis, $P=p^{A} \Sigma_{A}$. By Poincare duality there is a dual (1,1)-form $p^{A} \omega_{A}$, where $\omega_{A}$ belong to the basis of $H^{2}(X, \mathbb{Z})$ introduced above. The Kähler form of the internal threefold is proportional to $\operatorname{Im}\left(Y^{A} / Y^{0}\right) \omega_{A}$. Note that the stabilization equations (3.34) imply that the Kähler form, evaluated with the values that the Kähler moduli take at the horizon, is proportional to the Poincaré dual of $P$. If all $p^{A}$ are positive, the $(1,1)$-form $p^{A} \omega_{A}$ lays in the interior of the Käher cone and this provides a link between suppression of $\alpha^{\prime}$ corrections and $P$ being a very ample divisor. Namely, by multiplying the $(1,1)$-form with a large positive number or, equivalently, by taking the $p^{A}$ very large, we can arrange that $p^{A} \omega_{A}$ is far away from the boundaries of the Kähler cone. In terms of homology $p^{A}>0$ implies that the divisor $P$ has positive intersection numbers with all complex submanifolds of $X$. This shows that $P$ is a so-called ample divisor according to the Nakai-Moishezon criterion. By definition a divisor is called ample if it can be made very ample by multiplying it with a sufficiently large positive number. Thus $P$ is ample if $p^{A}>0$ and very ample if $p^{A} \gg 0$. As we explained above one has to require that $P$ is very ample in order to be able to reliably compute the microscopic entropy.

We now turn to the macroscopic entropy of a black hole with non-vanishing charges $q_{0}$ and $p^{A}$, which can be computed by substituting the function (3.33) into the general formula (1.1). In the case of a generic threefold the resulting expression for the macroscopic entropy fully agrees with the microscopic formula (3.30), as we already showed in [1]. For the case of a compactification on $K 3 \times T^{2}$ we find

$$
\mathscr{S}_{\text {macro }}=2 \pi \sqrt{\frac{1}{6}\left|q_{0}\right|\left(3 C_{a b} p^{a} p^{b} p^{1}+24 p^{1}\right)} .
$$

This too is in full agreement with the microscopic computation provided that the zero-mode counting for $K 3 \times T^{2}$ is modified as described above.

\section{A non-perturbative example}

Let us now consider an extension of the heterotic-like model of Section 2, where the term proportional to $\Upsilon$ is replaced by a more general function of $S=-i Y^{1} / Y^{0}$,

$$
F(Y, \Upsilon)=-\frac{Y^{1} Y^{a} \eta_{a b} Y^{b}}{Y^{0}}+F^{(1)}(S) \Upsilon,
$$


where $\eta_{a b}$ is defined as before. In Section 2 we noted that the dilaton and the black hole entropy depended only on combinations of the electric and magnetic charges that are invariant under classical (i.e. continuous) target-space duality transformations. For the extension discussed here, target-space duality remains realized, so we will first analyze this aspect in some more detail. Subsequently we will consider the possible invariance under $S$-duality.

First of all, the invariance under the $\mathrm{SO}(1, n-2)$ subgroup of the target-space duality group is manifest, as this is an invariance of (4.1). The transformations that extend this subgroup to the full $\mathrm{SO}(2, n-1)$ group of target-space dualities depend on $2 n-1$ parameters, which we write as two vectors $a_{a}$ and $b^{a}$ and a scalar $c$ under the subgroup. The charges $M_{I}$ and $N^{I}$ transform under the corresponding infinitesimal transformations according to

$$
\begin{array}{ll}
\delta M_{0}=c M_{0}+b^{a} M_{a}, & \delta N^{0}=-c N^{0}-a_{a} N^{a}, \\
\delta M_{1}=-c M_{1}-\frac{1}{2} \eta^{a b} a_{a} M_{b}, & \delta N^{1}=c N^{1}+2 \eta_{a b} b^{a} N^{b}, \\
\delta M_{a}=a_{a} M_{0}-2 \eta_{a b} b^{a} M_{1}, & \delta N^{a}=-b^{a} N^{0}+\frac{1}{2} \eta^{a b} a_{b} N^{1} .
\end{array}
$$

Using that the vectors $\left(Y^{0}, F_{1}, Y^{2}, \ldots, Y^{n}\right)$ and $\left(F_{0},-Y^{1}, F_{2}, \ldots, F_{n}\right)$ transform as $N^{I}$ and $M_{I}$ under target-space duality [34,35], the corresponding transformations of $Y^{I}$ and $F_{I}$ are given by

$$
\begin{array}{ll}
\delta Y^{0}=-c Y^{0}-a_{a} Y^{a}, & \delta F_{0}=c F_{0}+b^{a} F_{a}, \\
\delta Y^{1}=-c Y^{1}+\frac{1}{2} \eta^{a b} a_{a} F_{b}, & \delta F_{1}=c F_{1}+2 \eta_{a b} b^{a} Y^{b} \\
\delta Y^{a}=-b^{a} Y^{0}+\frac{1}{2} \eta^{a b} a_{b} F_{1}, & \delta F_{a}=a_{a} F_{0}+2 \eta_{a b} b^{a} Y^{1} .
\end{array}
$$

The crucial observation is now that $S$ is invariant under the above transformations of the $Y^{I}$, whereas these transformations induce the correct transformations on the derivatives $F_{I}$ as specified above. This means that the continuous $\mathrm{SO}(2, n-1)$ transformations are preserved for any function $F$ of the type given in (4.1). This explains the manifest target-space duality invariance of the expressions found in Section 2.

We now proceed and analyze the behaviour under $S$-duality. In general it is not known which subset of the $S$-duality transformations will be realized in $N=2$ heterotic string compactifications. But we consider this option, first as an example to appreciate the relevance of the non-holomorphic corrections for the entropy formula, and secondly with an eye towards the $N=4$ theory of which the $N=2$ theory is just a truncation. On the electric and the magnetic charges, the $S$-duality transformations act according to

$$
M_{I} \rightarrow \tilde{M}_{I}=a M_{I}-2 b \eta_{I J} N^{J}, \quad N^{I} \rightarrow \tilde{N}^{I}=d N^{I}-\frac{1}{2} c \eta^{I J} M_{J},
$$

where the parameters $a, b, c$ and $d$ are integers satisfying $a d-b c=1$, and where the symmetric matrix $\eta_{I J}$ and its inverse $\eta^{I J}$ are defined by

$$
\eta_{I J} N^{I} N^{J}=N^{0} N^{1}+\eta_{a b} N^{a} N^{b}, \quad \eta^{I J} M_{I} M_{J}=4 M_{0} M_{1}+\eta^{a b} M_{a} M_{b} .
$$

The above expressions are equal to two of the $\mathrm{SO}(2, n-1)$ invariants, $\frac{1}{2}\langle N, N\rangle$ and $2\langle M, M\rangle$, defined in (2.10). Under $S$-duality the three invariants transform according to

$$
\begin{aligned}
& \langle M, M\rangle \rightarrow a^{2}\langle M, M\rangle+b^{2}\langle N, N\rangle-2 a b M \cdot N, \\
& \langle N, N\rangle \rightarrow c^{2}\langle M, M\rangle+d^{2}\langle N, N\rangle-2 c d M \cdot N, \\
& M \cdot N \rightarrow-a c\langle M, M\rangle-b d\langle N, N\rangle+(a d+b c) M \cdot N .
\end{aligned}
$$


The quantities $Y^{I}$ and $F_{I}$ transform as

$$
\begin{array}{llrl}
Y^{0} \rightarrow \tilde{Y}^{0} & =d Y^{0}+c Y^{1}, & F_{0} & \rightarrow \tilde{F}_{0}=a F_{0}-b F_{1}, \\
Y^{1} \rightarrow \tilde{Y}^{1} & =a Y^{1}+b Y^{0}, & F_{1} & \rightarrow \tilde{F}_{1}=d F_{1}-c F_{0}, \\
Y^{a} \rightarrow \tilde{Y}^{a} & =d Y^{a}-\frac{1}{2} c \eta^{a b} F_{b}, & F_{a} & \rightarrow \tilde{F}_{a}=a F_{a}-2 b \eta_{a b} Y^{b} .
\end{array}
$$

As a result of these transformations one easily verifies that $S$ transforms according to the well-known SL(2) formula,

$$
S \rightarrow \tilde{S}=\frac{a S-i b}{i c S+d} .
$$

However, in this case the transformations of the $F_{I}$ are not, in general, correctly induced by the transformations of the $Y^{I}$. This is only the case when

$$
f(\tilde{S})=(i c S+d)^{2} f(S)
$$

where $f(S)=-i \partial F^{(1)}(S) / \partial S$. This implies that $f(S)$ must transform under $S$-duality transformations as a modular form of weight 2 .

In heterotic-like string compactifications, the Wilsonian coupling function $F^{(1)}(S)$ has an expansion of the type $F^{(1)}(S)=i c_{1} S+f(q)$, where $f(q)$ denotes a series expansion in positive powers of $q=\exp (-2 \pi S)$ and $c_{1}$ is the constant appearing in (2.1) (actually, in $N=2$ heterotic string compactifications the function $F$ will in general also receive perturbative corrections which depend on the moduli $T^{a}=-i Y^{a} / Y^{0}$. Here we do not discuss these perturbative corrections). It is well known that there is no such holomorphic $F^{(1)}(S)$ satisfying (4.9). Thus, there is no holomorphic Wilsonian coupling function consistent with $S$-duality. This, however, does not pose a problem, since in the presence of massless fields the Wilsonian coupling functions do not, in general, exhibit all the invariances of the physical effective coupling functions. In order to obtain a coupling function that is consistent with (4.9), we have to give up holomorphicity, which is a characteristic feature of Wilsonian couplings, and assume that $F^{(1)}$ depends both on $S$ and on $\bar{S}$. In doing so, we can still preserve the stabilization equations (where the $F_{I}$ remain given by $\partial F / \partial Y^{I}$ ) and moreover the (classical) target-space duality invariance remains intact. A non-holomorphic $F^{(1)}(S, \bar{S})$ which satisfies (4.9) and which in the weak coupling limit $\operatorname{Re} S \rightarrow \infty$ turns into $F^{(1)}(S, \bar{S}) \rightarrow i c_{1} S$ is given by $F^{(1)}(S, \bar{S})=-i c_{1}$ $\frac{6}{\pi}\left(\log \underline{\eta}^{2}(S)+\log (S+\bar{S})\right)$, so that $f(S, \bar{S})=-i \partial_{S} F^{(1)}(S, \bar{S})=c_{1} \frac{3}{\pi^{2}} G_{2}(S, \bar{S})$, where $G_{2}(S, \bar{S})=G_{2}(S)-2 \pi /(S+\bar{S})$ and $G_{2}(S)=-4 \pi \partial_{S} \log \eta(S)$ (see, e.g., Ref. [33]). We should stress here that $F^{(1)}$ is only determined up to an anti-holomorphic function of $\bar{S}$, which we suppressed in view of the fact that it should vanish for weak coupling. Thus, a function $F$ which is consistent with $S$-duality is given by $(4.1)$ with $F^{(1)}(S)$ replaced by $F^{(1)}(S, \bar{S})$. We note that $F^{(1)}(S, \bar{S})$ is not invariant under (4.8), but rather transforms as $F^{(1)}(S, \bar{S}) \rightarrow F^{(1)}(S, \bar{S})+i c_{1} \frac{6}{\pi} \log (-i c \bar{S}+d)$

Let us now turn to the entropy calculation using the same definitions for the stabilization equation and the entropy as before, but now with $F^{(1)}(S)$ replaced by $F^{(1)}(S, \bar{S})$. First we determine $|Z|^{2}=p^{I} F_{I}(Y, \bar{Y}, \Upsilon)-q_{I} Y^{I}$. By following the same steps as in Section 2 we obtain

$$
|Z|^{2}=(S+\bar{S})\left[\frac{1}{2}\langle N, N\rangle+(f(S, \bar{S}) \Upsilon+\text { h.c. })\right],
$$

which reduces to (2.6) in the weak coupling limit $\operatorname{Re} S \rightarrow \infty$. The value of the dilaton $S$ at the horizon is, in principle, determined in terms of the charges $M_{I}$ and $N^{I}$ carried by 
the black hole through the stabilization equations (1.4). Again, following the very same steps as in Section 2, we find

$$
\begin{aligned}
& S \bar{S}\langle N, N\rangle=\langle M, M\rangle-2(S+\bar{S})(f(S, \bar{S}) S \Upsilon+\text { h.c. }), \\
& (S-\bar{S})\langle N, N\rangle=2 i M \cdot N+2(S+\bar{S})(f(S, \bar{S}) \Upsilon-\text { h.c. }) .
\end{aligned}
$$

These two equations are combined into

$$
f(S, \bar{S}) \Upsilon=\frac{1}{4} \frac{1}{(S+\bar{S})^{2}}\left(M_{I}-2 i \bar{S} \eta_{I K} N^{K}\right) \eta^{I J}\left(M_{J}-2 i \bar{S} \eta_{J L} N^{L}\right) .
$$

Note that this equation should not be regarded as the solution for the function $f(S, \bar{S})$ but rather as an equation that determines $S$ in terms of the charges. It is clear that this equation is consistent with the required $S$-duality transformation of $f(S, \bar{S})$, since

$$
\begin{aligned}
& S+\bar{S} \rightarrow \frac{S+\bar{S}}{|i c S+d|^{2}}, \\
& M_{I}+2 i S \eta_{I K} N^{K} \rightarrow \frac{M_{I}+2 i S \eta_{I K} N^{K}}{i c S+d},
\end{aligned}
$$

under the transformations (4.4) and (4.8). Using (4.12) the expression for $|Z|^{2}$ takes a very simple form,

$$
|Z|^{2}=\frac{\left(M_{I}+2 i S \eta_{I K} N^{K}\right) \eta^{I J}\left(M_{J}-2 i \bar{S} \eta_{J L} N^{L}\right)}{2(S+\bar{S})}
$$

whose invariance under $S$-duality is manifest.

Using the result for $F^{(1)}$ with the non-holomorphic corrections included, one evaluates the expression for the entropy. As it turns out, the result is not invariant under $S$-duality, which forces us to include an extra term. Thus, not only does the symplectic vector $\left(Y^{I}, F_{I}(Y, \bar{Y}, \Upsilon)\right)$ receive non-holomorphic corrections, but there is an additional non-holomorphic correction to the coupling function of the $C_{\mu \nu \rho \sigma}^{2}$ term, with the result that its effective $S$-duality invariant coupling function is given by $F^{(1)}(S, \bar{S})+$ $i c_{1} \frac{3}{\pi} \log (S+\bar{S})$. The combined $S$ - and $T$-duality invariant expression for the entropy then reads (with $\Upsilon=-64$ )

$$
\begin{aligned}
\mathscr{S}_{\text {macro }}= & \pi\left[|Z|^{2}+4 \operatorname{Im}\left(\Upsilon F^{(1)}(S, \bar{S})+i c_{1} \frac{3}{\pi} \Upsilon \log (S+\bar{S})\right)\right] \\
= & \frac{\pi}{2} \frac{\left(M_{I}+2 i S \eta_{I K} N^{K}\right) \eta^{I J}\left(M_{J}-2 i \bar{S} \eta_{J L} N^{L}\right)}{S+\bar{S}} \\
& +768 c_{1} \log \left[(S+\bar{S})|\eta(S)|^{4}\right] .
\end{aligned}
$$

We should stress here that the first term does implicitly depend on the function $f(S, \bar{S})$ through the solution of (4.12) for $S$.

The above $N=2$ example can be viewed as describing an $N=2$ subsector of the effective Lagrangian of heterotic string theory compactified on a six-torus. We already discussed this truncation in Section 2. Hence (4.15) can be promoted to an $N=4$ target-space duality invariant result. This is accomplished by simply replacing the $\mathrm{SO}(2, n-1)$ invariants bilinear in the charges by the corresponding $\mathrm{SO}(6,22)$ ones. 
Indeed, it is known [33] that the effective Lagrangian of this $N=4$ theory possesses a $C_{\mu \nu \rho \sigma}^{2}$ term with an effective coupling function given by $F^{(1)}(S, \bar{S})+i c_{1} \frac{3}{\pi} \log (S+\bar{S})$. Since the $N=4$ theory is conjectured to be invariant under $S$-duality, the black hole entropy in the heterotic $N=4$ theory should also be invariant under strong-weak coupling duality. It would be interesting to make contact between the $N=4$ extension of (4.15) and the entropy formula of [36].

Eq. (4.12) for $S$ can be solved iteratively order-by-order in $\Upsilon$. In the case of purely imaginary $S$, the solution remains unmodified, that is $S=i M \cdot N /\langle N, N\rangle$.

\section{Corrections from T-dependent higher-derivative interaction terms}

So far we considered holomorphic functions $F(Y, \Upsilon)$ that depended at most linearly on $\Upsilon$. In this section we will study functions that depend more generally on $\Upsilon$, which we recall, is just proportional to the square of the auxiliary tensor field $T^{a b i j}$ which is the lowest component of the Weyl multiplet. More specifically, we will consider functions $F(Y, \Upsilon)$ associated with a type-IIA string compactification on a Calabi-Yau threefold. In the limit of large Kähler moduli, the corresponding homogeneous holomorphic function is given by

$$
F(Y, \Upsilon)=\frac{D_{A B C} Y^{A} Y^{B} Y^{C}}{Y^{0}}+d_{A} \frac{Y^{A}}{Y^{0}} \Upsilon+G\left(Y^{0}, \Upsilon\right)
$$

where $D_{A B C}=-\frac{1}{6} C_{A B C}, d_{A}=-\frac{1}{24} \frac{1}{64} c_{2 A}$. The coefficients $C_{A B C}$ denote the intersection numbers of the four-cycles of the Calabi-Yau threefold, whereas the coefficients $c_{2 A}$ denote its second Chern-class numbers. The function $G\left(Y^{0}, \Upsilon\right)$ is proportional to $[17,18]$

$$
I(\alpha)=\frac{\alpha^{2}}{4} \sum_{n \in \mathbb{Z}, n \neq 0} \int_{0}^{\infty} \frac{d s}{s} \frac{1}{\sinh ^{2}\left(\frac{1}{2} \alpha s\right)} \mathrm{e}^{-2 \pi i n s} .
$$

Here $\alpha$ is proportional to $\sqrt{\Upsilon} / Y^{0}$. As we shall argue below the proportionality factor relating $G\left(Y^{0}, \Upsilon\right)$ to $I(\alpha)$ is given by $G\left(Y^{0}, \Upsilon\right)=-i /\left(2(2 \pi)^{3}\right) \chi\left(Y^{0}\right)^{2} I(\alpha)$, where $\chi$ denotes the Euler number of the Calabi-Yau manifold. Observe that $G$ satisfies the homogeneity relation

$$
Y^{0} G_{0}+2 \Upsilon G_{\Upsilon}=2 G,
$$

where $G_{0}$ and $G_{\Upsilon}$ denote the derivatives of $G$ with respect to $Y^{0}$ and $\Upsilon$, respectively.

An explicit evaluation of the integral (5.2) has been given in two regimes $[17,18]$, namely one in which $\alpha$ is taken to be small and the other in which $\alpha$ is taken to be large. When computing (5.2) a regularisation prescription needs to be adopted. In the case of small $\alpha$, the regularised expression (5.2) reads [17,18,37]

$$
I(\alpha)_{\mathrm{reg}}=\sum_{g=0,2,3, \ldots} b_{g} \alpha^{2 g}, \quad b_{g}=(-)^{g} 2(2 g-1) \frac{\zeta(2 g) \zeta(3-2 g)}{(2 \pi)^{2 g}} .
$$

The coefficient $b_{0}$, in particular, is given by $b_{0}=-\zeta(3)$, which shows that the proportionality factor relating $G\left(Y^{0}, \Upsilon\right)$ to $I(\alpha)_{\text {reg }}$ must be the one mentioned above, since the function $F$ is known to contain a term $i /\left(2(2 \pi)^{3}\right) \zeta(3) \chi\left(Y^{0}\right)^{2}$ [38]. We note 
that, for small $\alpha$, the function $G\left(Y^{0}, \Upsilon\right)$ is not actually fully captured by (5.4) since there are additional contributions to it which are not analytic near $\alpha=0[17,18]$.

In a number of cases one can perform explicit calculations and determine the value of the scalar fields $Y^{I}$ (from (1.2)) and of the entropy (from (1.1)) in the presence of a function $G\left(Y^{0}, \Upsilon\right)$. Below we will evaluate the case with $p^{0}=0$ (so that $Y^{0}$ is real), the case of axion-free black holes and the case where $Y^{0}$ is imaginary and thus equal to $\frac{1}{2} i p^{0}$. To facilitate this discussion we first collect a number of useful formulae.

First of all, the scalars $Y^{A}$ do not appear in the function $G$, which implies that

$$
F_{A}=\frac{3 D_{A B C} Y^{B} Y^{C}+d_{A} \Upsilon}{Y^{0}}
$$

remains unchanged. Therefore the stabilization equations for $q_{A}$ are universal (since these equations are only valid at the horizon, we have $\Upsilon=-64$ ),

$$
q_{A}=\frac{1}{\left|Y^{0}\right|^{2}}\left[-d_{A} p^{0} \Upsilon-3 i D_{A B C}\left(Y^{B} Y^{C} \bar{Y}^{0}-\bar{Y}^{B} \bar{Y}^{C} Y^{0}\right)\right] \text {. }
$$

The stabilization equation for $q_{0}$ does depend on the function $G$ and reads

$$
q_{0}=i \frac{D_{A B C} Y^{A} Y^{B} Y^{C}+d_{A} Y^{A} \Upsilon}{\left(Y^{0}\right)^{2}}-i \frac{D_{A B C} \bar{Y}^{A} \bar{Y}^{B} \bar{Y}^{C}+d_{A} \bar{Y}^{A} \bar{\Upsilon}}{\left(\bar{Y}^{0}\right)^{2}}-i\left(G_{0}-\bar{G}_{0}\right) .
$$

Furthermore, the expression for $|Z|^{2}=p^{I} F_{I}-q_{I} Y^{I}$ can be written as follows:

$$
\begin{aligned}
|Z|^{2}= & i D_{A B C}\left[\frac{3 Y^{A} Y^{B} \bar{Y}^{C}}{Y^{0}}-\frac{3 \bar{Y}^{A} \bar{Y}^{B} Y^{C}}{\bar{Y}^{0}}-\frac{Y^{A} Y^{B} Y^{C} \bar{Y}^{0}}{\left(Y^{0}\right)^{2}}+\frac{\bar{Y}^{A} \bar{Y}^{B} \bar{Y}^{C} Y^{0}}{\left(\bar{Y}^{0}\right)^{2}}\right] \\
& +i d_{A}\left[\frac{\bar{Y}^{A} \Upsilon}{Y^{0}}-\frac{Y^{A} \bar{\Upsilon}}{\bar{Y}^{0}}-\frac{Y^{A} \bar{Y}^{0} \Upsilon}{\left(Y^{0}\right)^{2}}+\frac{\bar{Y}^{A} Y^{0} \bar{\Upsilon}}{\left(\bar{Y}^{0}\right)^{2}}\right]+\frac{1}{2} i\left(Y^{0}+\bar{Y}^{0}\right)\left(G_{0}-\bar{G}_{0}\right) \\
& +\frac{1}{2} p^{0}\left(G_{0}+\bar{G}_{0}\right) .
\end{aligned}
$$

The entropy is given by

$$
\mathscr{S}_{\text {macro }}=\pi\left[|Z|^{2}-2 i d_{A}\left(\frac{Y^{A}}{Y^{0}} \Upsilon-\frac{\bar{Y}^{A}}{\bar{Y}^{0}} \bar{\Upsilon}\right)-2 i\left(\Upsilon G_{\Upsilon}-\bar{\Upsilon} \bar{G}_{\Upsilon}\right)\right] .
$$

We now turn to the three separate cases.

\subsection{Black holes with $p^{0}=0$}

Let us compute the entropy for a class of type-IIA black holes with $p^{0}=0$. This implies immediately that $Y^{0}=\bar{Y}^{0}$. Eq. (5.6) then simplifies and one establishes directly that

$$
Y^{A}=\frac{1}{6} Y^{0} D^{A B} q_{B}+\frac{1}{2} i p^{A}, \quad \text { where } \quad D_{A B} \equiv D_{A B C} p^{C}, \quad D_{A B} D^{B C}=\delta_{A}^{C},
$$


where we recall that $D_{A B C}$ and $d_{A}$ are related to the four-cycle intersection numbers and the second Chern-class numbers of an underlying Calabi-Yau manifold. Similarly, one obtains from (5.7),

$$
4\left(Y^{0}\right)^{2}=\frac{D_{A B C} p^{A} p^{B} p^{C}-4 d_{A} p^{A} \Upsilon}{\hat{q}_{0}+i\left(G_{0}-\bar{G}_{0}\right)},
$$

where $\hat{q}_{0} \equiv q_{0}+\frac{1}{12} D^{A B} q_{A} q_{B}$. Furthermore, from (5.8) we determine

$$
|Z|^{2}=-\frac{D_{A B C} p^{A} p^{B} p^{C}-2 d_{A} p^{A} \Upsilon}{Y^{0}}+i Y^{0}\left(G_{0}-\bar{G}_{0}\right) \text {. }
$$

Combining these results leads to the following equation for the entropy:

$$
\mathscr{S}_{\text {macro }}=-4 \pi Y^{0} \hat{q}_{0}-i \pi\left(3 Y^{0} G_{0}+2 \Upsilon G_{\Upsilon}-\text { h.c. }\right) \text {. }
$$

For vanishing $G$ this result agrees with that given in [1], which was consistent with the results of state counting presented in [2,3] in appropriate limits.

Eq. (5.11) can be solved iteratively for $Y^{0}$ in terms of the charges, as follows. Let us denote the value of $Y^{0}$ by $y^{0}$ when $G$ is switched off. We take the magnetic charges $p^{A}$ to be positive and $\hat{q}_{0}<0$, so that $\left(y^{0}\right)^{2}>0$, as can be seen from (5.11). In a regime where $\left|\operatorname{Im} G_{0}\left(y^{0}, \Upsilon\right)\right| \ll\left|\hat{q}_{0}\right|,(5.11)$ can, to first approximation, be written as

$$
Y^{0}=y^{0}\left(1+\frac{1}{2} \frac{i\left(G_{0}\left(y^{0}, \Upsilon\right)-\bar{G}_{0}\left(\bar{y}^{0}, \bar{\Upsilon}\right)\right)}{\left|\hat{q}_{0}\right|}+\ldots\right) .
$$

Inserting (5.14) into (5.13) then yields, to first approximation,

$$
\begin{aligned}
\mathscr{S}_{\text {macro }}= & 2 \pi \sqrt{\frac{1}{6}\left|\hat{q}_{0}\right|\left(C_{A B C} p^{A} p^{B} p^{C}+c_{2 A} p^{A}\right)} \\
& -2 \pi i\left(G\left(y^{0}, \Upsilon\right)-\bar{G}\left(\bar{y}^{0}, \bar{\Upsilon}\right)\right)+\cdots,
\end{aligned}
$$

where we used the homogeneity property (5.3) for $G$ and expressed the result in terms of the intersection numbers and second Chern-class numbers.

\subsection{Axion-free black holes}

Now let us consider axion-free black holes, which are characterized by the fact that the moduli $z^{A}=Y^{A} / Y^{0}$ are imaginary. Using $\bar{Y}^{0} Y^{A}+Y^{0} \bar{Y}^{A}=0$, it readily follows that

$$
Y^{A}=i p^{A} \frac{Y^{0}}{\lambda}, \quad \text { where } \quad Y^{0}=\frac{1}{2}\left(\lambda+i p^{0}\right) .
$$

The charges $q_{A}$ follow directly from (5.6),

$$
q_{A}=-3 D_{A B C} p^{B} p^{C} \frac{p^{0}}{\lambda^{2}}-4 d_{A} \frac{p^{0} \Upsilon}{\lambda^{2}+\left(p^{0}\right)^{2}} .
$$


This equation implies that the charges are tightly constrained. It also implies a quadratic equation for $\lambda^{2}$. Its solution thus fixes all the moduli in terms of the charges. The stabilization Eq. (5.7) for $q_{0}$ shows that $q_{0}$ is not independent, but given by

$$
q_{A} p^{A}+3 q_{0} p^{0}=-d_{A} p^{A} \Upsilon \frac{16 p^{0}}{\lambda^{2}+\left(p^{0}\right)^{2}}-3 i p^{0}\left(G_{0}-\bar{G}_{0}\right) \text {. }
$$

In this case (5.8) yields

$$
\begin{aligned}
|Z|^{2}= & -2 D_{A B C} p^{A} p^{B} p^{C} \frac{\lambda^{2}+\left(p^{0}\right)^{2}}{\lambda^{3}}+4 d_{A} p^{A} \Upsilon \frac{\lambda^{2}-\left(p^{0}\right)^{2}}{\lambda\left(\lambda^{2}+\left(p^{0}\right)^{2}\right)} \\
& +\frac{1}{2} i \lambda\left(G_{0}-\bar{G}_{0}\right)+\frac{1}{2} p^{0}\left(G_{0}+\bar{G}_{0}\right) .
\end{aligned}
$$

The entropy can be written as follows (with $\Upsilon=-64$ ):

$$
\begin{aligned}
\mathscr{S}_{\text {macro }}=\pi[ & -2 D_{A B C} p^{A} p^{B} p^{C} \frac{\lambda^{2}+\left(p^{0}\right)^{2}}{\lambda^{3}}+8 d_{A} p^{A} \Upsilon \frac{\lambda}{\lambda^{2}+\left(p^{0}\right)^{2}} \\
& \left.+\frac{1}{2} i \lambda\left(G_{0}-\bar{G}_{0}\right)+\frac{1}{2} p^{0}\left(G_{0}+\bar{G}_{0}\right)-2 i \Upsilon\left(G_{\Upsilon}-\bar{G}_{\Upsilon}\right)\right] .
\end{aligned}
$$

For $G=i c\left(Y^{0}\right)^{2}$ and $d_{A}=0$ this result coincides with that obtained in [24].

5.3. Black holes with $\operatorname{Re} Y^{0}=0$

Finally, we consider black holes for which $\operatorname{Re} Y^{0}=0$ and, hence, $Y^{0}=\frac{1}{2} i p^{0}$. The stabilization equations (5.6) and (5.7) yield the following two conditions:

$$
\begin{gathered}
p^{0} q_{A}=-6 D_{A B C}\left(Y^{B} Y^{C}+\bar{Y}^{B} \bar{Y}^{C}\right)-4 d_{A} \Upsilon, \\
\left(p^{0}\right)^{2} q_{0}=4 D_{A B C} p^{A}\left(Y^{B} Y^{C}+Y^{B} \bar{Y}^{C}+\bar{Y}^{B} \bar{Y}^{C}\right) \\
+4 d_{A} p^{A} \Upsilon-i\left(p^{0}\right)^{2}\left(G_{0}-\bar{G}_{0}\right) .
\end{gathered}
$$

Just as in the previous subsection the charge $q_{0}$ is not independent and is constrained by

$$
p^{0} p^{I} q_{I}=2 D_{A B C} p^{A} p^{B} p^{C}-i\left(p^{0}\right)^{2}\left(G_{0}-\bar{G}_{0}\right) .
$$

Eqs. (5.21) are quadratic in terms of the scalars $Y^{A}$ which can therefore be determined in terms of the charges. However, we do not wish to pursue this in full generality. Below we will determine the value of the $Y^{A}$ for type-II models with a dual heterotic description.

From (5.8) and (5.9) we obtain

$$
\begin{aligned}
|Z|^{2}= & \frac{2}{p^{0}} D_{A B C}(Y+\bar{Y})^{A}(Y+\bar{Y})^{B}(Y+\bar{Y})^{C}+\frac{4}{p^{0}} d_{A}(Y+\bar{Y})^{A} \Upsilon \\
& +\frac{1}{2} p^{0}\left(G_{0}+\bar{G}_{0}\right), \\
\mathscr{S}_{\text {macro }}= & \frac{2 \pi}{p^{0}} D_{A B C}(Y+\bar{Y})^{A}(Y+\bar{Y})^{B}(Y+\bar{Y})^{C}-2 i \pi(G-\bar{G}),
\end{aligned}
$$

where we made use of the homogeneity property (5.3) for $G$. 
For type-II models with a dual heterotic description, we can use the first equation in (5.21) to determine the $Y^{A}$, along the same lines as in Section 2. We start from

$$
D_{A B C} Y^{A} Y^{B} Y^{C}=-Y^{1} Y^{a} \eta_{a b} Y^{b}, \quad d_{A} Y^{A}=c_{1} Y^{1},
$$

so that $D_{1 a b}=-\frac{1}{3} \eta_{a b}$. Then the first equation in (5.21) yields

$$
\begin{aligned}
Y^{a}=\frac{1}{Y^{1}+\bar{Y}^{1}}\left[\frac{1}{4} p^{0} \eta^{a b} q_{b}\right. & \left.+i \bar{Y}^{1} p^{a}\right], \\
4 c_{1} \Upsilon+p^{0} q_{1}+p^{a} \eta_{a b} p^{b}= & \frac{1}{\left(Y^{1}+\bar{Y}^{1}\right)^{2}}\left[\frac{1}{4}\left(p^{0}\right)^{2} q_{a} \eta^{a b} q_{b}+\left(p^{1}\right)^{2} p^{a} \eta_{a b} p^{b}\right. \\
& \left.+p^{0} p^{1} p^{a} q_{a}\right] .
\end{aligned}
$$

Substituting this into the above entropy formula, we obtain

$$
\begin{aligned}
\mathscr{S}_{\text {macro }}= & -\frac{2 \pi}{p^{0}\left(Y^{1}+\bar{Y}^{1}\right)}\left[\frac{1}{4}\left(p^{0}\right)^{2} q_{a} \eta^{a b} q_{b}+\left(p^{1}\right)^{2} p^{a} \eta_{a b} p^{b}+p^{0} p^{1} p^{a} q_{a}\right] \\
& -2 i \pi(G-\bar{G}) \\
= & -\frac{\pi}{p^{0}} \sqrt{\left(\left(p^{0}\right)^{2} q_{a} \eta^{a b} q_{b}+4\left(p^{1}\right)^{2} p^{a} \eta_{a b} p^{b}+4 p^{0} p^{1} q_{a} p^{a}\right)} \\
& \times \sqrt{\left(q_{1} p^{0}+p^{a} \eta_{a b} p^{b}+4 c_{1} \Upsilon\right)}-2 i \pi(G-\bar{G}),
\end{aligned}
$$

We thus see that we have to choose $p^{0}<0$. This expression can be rewritten as follows in terms of the heterotic electric and magnetic charges $M_{I}$ and $N^{I}$ given in Section 2,

$$
\begin{aligned}
\mathscr{S}_{\text {macro }}= & -\frac{\pi}{p^{0}} \sqrt{\left(\left(p^{0}\right)^{2}\langle M, M\rangle+\left(p^{1}\right)^{2}\langle N, N\rangle+2 p^{0} p^{1} M \cdot N\right)} \\
& \times \sqrt{\left(\langle N, N\rangle+8 c_{1} \Upsilon\right)}-2 i \pi(G-\bar{G}) .
\end{aligned}
$$

Observe that the charges are subject to the constraint (5.22), which in the case at hand reads $p^{0} M \cdot N+p^{1}\langle N, N\rangle=-i\left(p^{0}\right)^{2}\left(G_{0}-\bar{G}_{0}\right)$. Substituting this into (5.27) yields

$$
\begin{aligned}
\mathscr{S}_{\text {macro }}= & \pi \sqrt{\langle M, M\rangle\langle N, N\rangle-(M \cdot N)^{2}-\left(p^{0}\right)^{2}\left(G_{0}-\bar{G}_{0}\right)^{2}} \\
& \times \sqrt{1-\frac{512 c_{1}}{\langle N, N\rangle}-2 i \pi(G-\bar{G}),}
\end{aligned}
$$

where we also used $\Upsilon=-64$. This expression reduces to (2.9) in the case of $G=0$.

\section{Note added}

It should be noted that the revised counting of micro-states proposed in Section 3 yields results that are consistent with anomaly inflow arguments [39]. We would like to thank J.A. Harvey and F. Larsen for discussions concerning this point. 


\section{Acknowledgements}

We thank K. Behrndt, R. Dijkgraaf, D. Lüst and E. Verlinde for valuable discussions. We also thank B. Hunt for clarifying discussions on the mathematical aspects of Section 3. G.L.C. thanks the CBPF in Rio de Janeiro for kind hospitality during the final stages of this work. B.d.W thanks the Alexander von Humboldt-Stiftung for supporting his stay at the AEI as part of the Humboldt Award Program.

\section{References}

[1] G.L. Cardoso, B. de Wit, T. Mohaupt, Phys. Lett. B 451 (1999) 309, hep-th/9812082.

[2] J.M. Maldacena, A. Strominger, E. Witten, J. High Energy Phys. 12 (1997) 2, hep-th/9711053.

[3] C. Vafa, Adv. Theor. Math. Phys. 2 (1998) 207, hep-th/9711067.

[4] G. Gibbons, in Supersymmetry, Supergravity and Related Topics, ed. F. del Aguila, J. de Azcárraga, L. Ibánẽz (World Scientific, Singapore, 1985) p. 147.

[5] R.M. Wald, Phys. Rev. D 48 (1993) 3427, gr-qc/9307038.

[6] T. Jacobson, G. Kang, R.C. Myers, Phys. Rev. D 49 (1994) 6587, gr-qc/9312023.

[7] V. Iyer, R.M. Wald, Phys. Rev. D 50 (1994) 846, gr-qc/9403028.

[8] T. Jacobson, G. Kang, R.C. Myers, Black-hole entropy in higher curvature gravity, gr-qc/9502009.

[9] G.L. Cardoso, B. de Wit, T. Mohaupt, Deviations from the area law for supersymmetric black holes, hep-th/9904005.

[10] S. Ferrara, R. Kallosh, A. Strominger, Phys. Rev. D 52 (1995) 5412, hep-th/9508072.

[11] A. Strominger, Phys. Lett. B 383 (1996) 39, hep-th/9602111.

[12] S. Ferrara, R. Kallosh, Phys. Rev. D 54 (1996) 1514, hep-th/9602136.

[13] S. Ferrara, R. Kallosh, Phys. Rev. D 54 (1996) 1525, hep-th/9603090.

[14] G. Moore, Attractors and Arithmetic, hep-th/9807056.

[15] G. Moore, Arithmetic and Attractors, hep-th/9807087.

[16] K. Behrndt, G.L. Cardoso, B. de Wit, D. Lüst, T. Mohaupt, W.A. Sabra, Phys. Lett. B 429 (1998) 289, hep-th/9801081.

[17] R. Gopakumar, C. Vafa, M-Theory and Topological Strings-I, hep-th/9809187.

[18] R. Gopakumar, C. Vafa, M-Theory and Topological Strings-II, hep-th/9812127.

[19] M. Bershadsky, S. Cecotti, H. Ooguri, C. Vafa, Nucl. Phys. B 405 (1993) 279, hep-th/9302103.

[20] M. Bershadsky, S. Cecotti, H. Ooguri, C. Vafa, Comm. Math. Phys. 165 (1994) 311, hep-th/9309140.

[21] I. Antoniadis, E. Gava, K.S. Narain, T.R. Taylor, Nucl. Phys. B 413 (1994) 162, hep-th/9307158.

[22] B. de Wit, Nucl. Phys. (Proc. Suppl.) B 49 (1996) 191, hep-th/9602060.

[23] B. de Wit, Fortsch. Phys. 44 (1996) 529, hep-th/9603191.

[24] K. Behrndt, G.L. Cardoso, B. de Wit, R. Kallosh, D. Lüst, T. Mohaupt, Nucl. Phys. B 488 (1997) 236, hep-th/9610105.

[25] G.L. Cardoso, D. Lüst, T. Mohaupt, Phys. Lett. B 388 (1996) 266, hep-th/9608099.

[26] P. Griffith, J. Harris, Principles of Algebraic Geometry (Wiley, New York, 1978).

[27] W. Barth, C. Peters, A. Van de Ven, Compact Complex Surfaces (Springer, Berlin, 1980).

[28] T. Hübsch, Calabi-Yau Manifolds (World Scientific, Singapore, 1992).

[29] P. Candelas, Lectures on Complex Manifolds, Lectures Notes of Trieste Spring School 1987.

[30] K. Becker, M. Becker, A. Strominger, Nucl. Phys. B 456 (1995) 130, hep-th/9507158.

[31] P.S. Aspinwall, D.R. Morrison, Mirror Symmetry II, ed. B. Greene, S.-T. Yau (International Press, 1997) p. 703, hep-th/9404151.

[32] M. Bershadsky, V. Sadov, C. Vafa, Nucl. Phys. B 463 (1996) 420, hep-th/9511222.

[33] J.A. Harvey, G. Moore, Phys. Rev. D 57 (1998) 2323, hep-th/9610237.

[34] A. Ceresole, R. D’Auria, S. Ferrara, A. Van Proeyen, Nucl. Phys. B 444 (1995) 92, hep-th/9502072.

[35] B. de Wit, V. Kaplunovsky, J. Louis, D. Lüst, Nucl. Phys. B 451 (1995) 53, hep-th/9504006.

[36] R. Dijkgraaf, E. Verlinde, H. Verlinde, Nucl. Phys. B 484 (1997) 543, hep-th/9607026.

[37] M. Mariño, G. Moore, Nucl. Phys. B 543 (1999) 592, hep-th/9808131.

[38] S. Hosono, A. Klemm, S. Theisen, S.-T. Yau, Nucl. Phys. B 433 (1995) 501, hep-th/9406055.

[39] J.A. Harvey, R. Minasian, G. Moore, J. High Energy Phys. 9 (1998) 4, hep-th/9808060. 